\title{
Internal decoupling in non-linear process control
}

\section{JENS G. BALCHEN $\dagger$ BERNT LIE $\dagger$ and INGAR SOLBERG $\dagger$}

Keywords: Non-linear control; linearization by transformation; multi-variable control; stability; robustness analysis.

\begin{abstract}
A simple method has been investigated for the total or partial removal of the effect of non-linear process phenomena in multi-variable feedback control systems. The method is based upon computing the control variables which will drive the process at desired rates. It is shown that the effect of model errors in the linearization of the process can be partly removed through the use of large feedback gains. In practice there will be limits on how large gains can be used. The sensitivity to parameter errors is less pronounced and the transient behaviour is superior to that of ordinary PI controllers.
\end{abstract}

\section{Introduction}

The model

$$
\dot{x}=f(x, u, v)
$$

describes a process where

$$
\begin{array}{ll}
x: & \text { state vector } \\
\boldsymbol{u}: & \text { control vector } \\
\boldsymbol{v}: & \text { disturbance vector }
\end{array}
$$

If a constant gain feedback control system is closed from $x$ to $\boldsymbol{u}$, the non-linear function $f(\cdot)$ will appear in the closed loop gain and will limit the performance in terms of stability etc.

It is therefore desirable to design a non-linear series compensator (decoupler) which tends to linearize the loop. This is done by computing a value of $\boldsymbol{u}$ which will drive the process at a desired rate $\dot{\boldsymbol{x}}_{\mathrm{d}}$, which can be referred to as a transformed control variable. This is equivalent to finding a decoupling transfer matrix in the linear case which makes the loop transfer matrix into a diagonal matrix of integrators.

Similar problems have been investigated by a number of authors, see Juba and Hamer (1986).

Setting

$$
\dot{x}_{\mathrm{d}}=f(x, u, v)
$$

and solving for $\boldsymbol{u}$ yields

$$
u=h\left(x, \dot{x}_{\mathrm{d}}, v\right)
$$

assuming that this solution exists.

Received 15 September 1987.

$\dagger$ Division of Engineering Cybernetics, Norwegian Institute of Technology, N-7034 Trondheim-NTH, Norway.

This paper was presented at the 10th IFAC World Congress on Automatic Control, Munich, West-Germany, July 27-31 1987 and is reprinted with the permission of IFAC. 


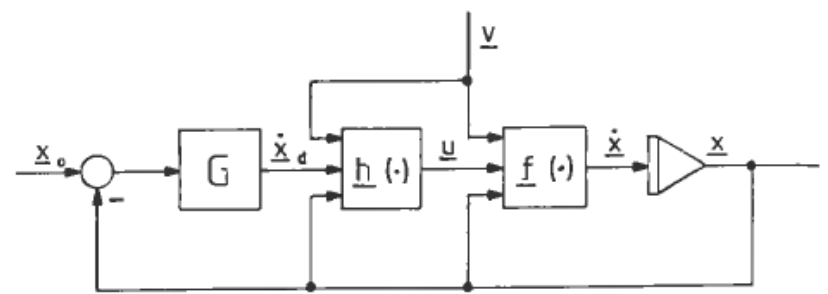

Figure 1. Structure of the internal decoupling scheme.

A common structure of the process model is

$$
\dot{x}=a(x, v)+B(x, v) \cdot u
$$

which is particularly convenient for the present purpose. This situation is quite common in chemical processes where energy and mass balances are involved. Both these kinds of balance equations are often linear in terms of the control variables such as added energy and flow rates. If (4) is valid, the control vector is particularly easy to find,

$$
u=B^{-1}(x, v) \cdot\left[\dot{x}_{\mathrm{d}}-a(x, v)\right]
$$

assuming that $B^{-1}(\cdot)$ exists.

As we have presupposed, applying the ideal solution (3) to (1) gives

$$
\dot{\boldsymbol{x}}=\dot{\boldsymbol{x}}_{\mathrm{d}}
$$

Deriving the transformed control variable $\dot{\boldsymbol{x}}_{\mathrm{d}}$ by the linear feedback control law

$$
\dot{x}_{d}=G\left(x_{0}-x\right)
$$

(where $G$ is a constant gain matrix or a multi-variable PI controller), will give a system like that in Fig. 1.

Equations (6) and (7) indicate that the consequence of assuming ideal conditions is that the non-linear effects of the process and the influence of the disturbances have disappeared. Here the matrix $G$ can be made diagonal and its gain elements are selected to be as large as is required to make the control system perform well. A similar technique has become popular in robotic control, where it is referred to as 'the computed torque technique', (Paul (1972).)

Complete linearization of (1) by (3) is based upon several ideal assumptions

(a) The process model

$$
\dot{x}=f_{\mathrm{m}}(x, u, v)
$$

describes the real process perfectly, i.e. the process model is known exactly.

(b) There is a unique solution $\boldsymbol{u}$ to (1) for arbitrary $\boldsymbol{x}, \dot{\boldsymbol{x}}$ and $\boldsymbol{v}$. This means, among other things, that it is necessary that $\operatorname{dim} u=\operatorname{dim} x$.

(c) The disturbance $v$ is known exactly.

(d) The state vector $\boldsymbol{x}$ is completely available, and not just a measurement vector $\boldsymbol{y}$ described by

$$
\boldsymbol{y}=\boldsymbol{g}(\boldsymbol{x})
$$

where $\operatorname{dim} \boldsymbol{y}<\operatorname{dim} \boldsymbol{x}$. 


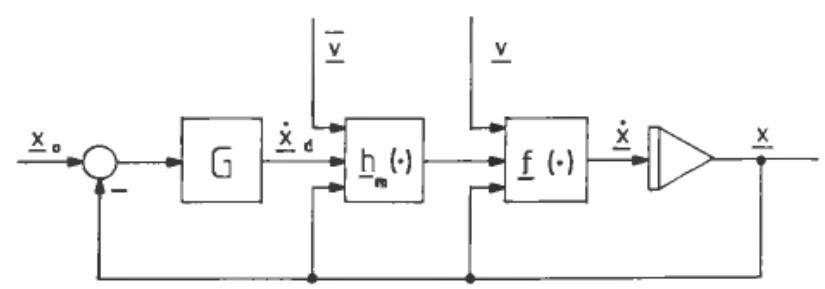

Figure 2. Non-linear decoupling with an error in the model.

The assumption in $(b)$ is not strictly necessary. Under certain conditions it is possible to find mappings transforming the state variables and the control variables into a transformed, linear system, even when $\operatorname{dim} u<\operatorname{dim} x$, see e.g. Hunt, Su and Meyer (1983). These transformations will not be discussed further in this present paper.

A violation of the assumption in $(c)$ is no more severe than in any other feedback control system with non-measurable process disturbances. A violation of the assumption in $(d)$ can be solved by introducing a state estimator which has to use the non-linear model (1) or (8). There will be no further discussion of these two cases in this paper.

Attention will now be turned to the violation of the assumptions in (a) and (b). In $\S 2$ we deal with controllers based upon a model deviating from the real process. The effect of this on a linear system is analysed. In $\S 3$, we address the case where $\operatorname{dim} u<\operatorname{dim} x$. Section 4 contains a discussion on how to solve (2) by using a gradient technique. In $\S 5$, we simulate a simple second order chemical reactor with one control variable in order to study the effects of model error on the control of the reactor.

\section{The case of an error in the process model}

First it is assumed that the inverse (3) exists, but that it is based upon the model (8). We will therefore have

$$
u=h_{\mathrm{m}}\left(x, \dot{x}_{\mathrm{d}}, \bar{v}\right)
$$

instead of (3), or

$$
\boldsymbol{u}=\tilde{h}_{\mathrm{m}}\left(x, \dot{x}_{\mathrm{d}}, \bar{v}\right)
$$

if we approximate (10).

The solution now becomes that illustrated in Fig. 2. Obviously we will have

$$
\begin{aligned}
\dot{x} & =f(x, u, v)=f\left(x, h_{\mathrm{m}}\left(x, \dot{x}_{\mathrm{d}}, \bar{v}\right), v\right) \\
& =\tilde{f}\left(x, \dot{x}_{\mathrm{d}}, \bar{v}, v\right)
\end{aligned}
$$

where $\tilde{f}$ is no longer equal to $\dot{\boldsymbol{x}}_{\mathrm{d}}$, but rather to a new non-linear mapping. We now wish to analyse how the system in Fig. 2 performs particularly with reference to stability. The easiest situation which reveals most of the structure of the solution is the linear case.

We assume the system to be

$$
\dot{x}=A x+B u+C v
$$




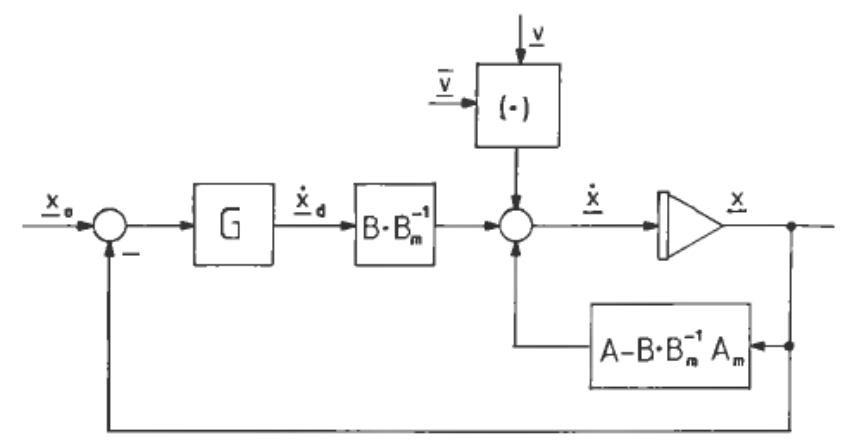

Figure 3. Decoupling of a linear system with model errors.

and that we use the model

$$
\dot{\boldsymbol{x}}=A_{\mathrm{m}} \boldsymbol{x}+B_{\mathrm{m}} u+C_{\mathrm{m}} \bar{v}
$$

From (14) we get analogous to (5)

$$
\boldsymbol{u}=B_{\mathrm{m}}^{-1}\left(\dot{\boldsymbol{x}}_{\mathrm{d}}-A_{\mathrm{m}} \boldsymbol{x}-C_{\mathrm{m}} \overline{\boldsymbol{v}}\right)
$$

which when applied to (13) will give

$$
\dot{\boldsymbol{x}}=\left(A-B B_{\mathrm{m}}^{-1} A_{\mathrm{m}}\right) x+B B_{\mathrm{m}}^{-1} \dot{x}_{\mathrm{d}}-B B_{\mathrm{m}}^{-1} C_{\mathrm{m}} \bar{v}+C \boldsymbol{v}
$$

In (16), the term

$$
\Delta A=A-B B_{\mathrm{m}}^{-1} A_{\mathrm{m}}
$$

should ideally have been $\Delta A=0$. However, in order for this to occur we need both $A_{\mathrm{m}}=A$ and $B_{\mathrm{m}}=B$. The total feedback control solution using (7) is shown in Fig. 3.

With reference to the stability of the loop, the important expression is

$$
\dot{\boldsymbol{x}}=\left(A-B B_{\mathrm{m}}^{-1} A_{\mathrm{m}}-B B_{\mathrm{m}}^{-1} G\right) \boldsymbol{x}=-\tilde{G} \boldsymbol{x}
$$

The eigenvalues of $\tilde{G}$ determine the system stability. Rewriting (18) we get

$$
-\tilde{G}=\left[\left(I-B B_{\mathrm{m}}^{-1} A_{\mathrm{m}} A^{-1}\right) A G^{-1}-B B_{\mathrm{m}}^{-1}\right] G
$$

The terms $A_{\mathrm{m}} A^{-1}$ and $B B_{\mathrm{m}}^{-1}$ should ideally be equal to $I$. Introducing

$$
\begin{aligned}
A_{\mathrm{m}} & =\left(I+E_{\mathrm{A}}\right) A \\
B_{\mathrm{m}}^{-1} & =B^{-1}\left(I+E_{\mathrm{B}}\right)
\end{aligned}
$$

where $E_{\mathrm{A}}$ and $E_{\mathrm{B}}$ are matrices expressing the relative errors in the modeling of $A$ and $B^{-1},(19)$ will become

$$
\begin{aligned}
\tilde{G} & =\left[\left(E_{\mathrm{A}}+E_{\mathrm{B}}+E_{\mathrm{B}} \cdot E_{\mathrm{A}}\right) A G^{-1}+E_{\mathrm{a}}\right] G+G \\
& \approx\left[\left(E_{\mathrm{A}}+E_{\mathrm{B}}\right) A G^{-1}+E_{\mathrm{B}}\right] G+G
\end{aligned}
$$

where it is assumed that the errors $E_{\mathrm{A}}$ and $E_{\mathrm{B}}$ are relatively small. Equation (22) clearly shows that the relative change in $G$ due to modeling errors consists of two terms, $\left(E_{\mathrm{A}}+E_{\mathrm{B}}\right) A G^{-1}$ and $E_{\mathrm{B}}$. The first term is highly dependent on the magnitude of $A G^{-1}$. If the elements of $G$ have large values (large gains) relative to the values of $A$, the first term will become insignificant. Consequently the most significant part of (22) is the term $E_{\mathrm{B}}$ expressing the error in $B^{-1}$. 
We can choose to let $G$ be diagonal. Hence, the elements of $G$ are the eigenvalues of the system when we have a perfect model. If the elements of $G$ have large values, (22) becomes

$$
\widetilde{G} \approx\left(I+E_{\mathrm{B}}\right) G
$$

where $E_{B}$ now expresses the relative shift in the eigenvalues of the loop depending on the error in modeling $B^{-1}$. This means that the system can tolerate relatively large errors $E_{\mathrm{B}}$ before the system becomes unstable.

Using (16), (19), (20) and (21), the final value theorem shows that the response in $x$ to a step change in $\boldsymbol{x}_{0}$ will be

$$
x=\tilde{G}^{-1}\left(I+E_{\mathrm{B}}\right) G x_{0}
$$

Thus, if the elements of $G$ are large enough to make (23) valid, there will only be a small stationary deviation due to a step change.

On the other hand it may also be of interest to use a multivariable PI controller instead of $G$, thereby removing any steady state error.

\section{The case of few control variables}

We will now consider the case of where

$$
\operatorname{dim} u<\operatorname{dim} x
$$

Here it is only possible to control the rate of a certain number of the system's states. In the linear case we find

$$
\left[\begin{array}{c}
\dot{x}_{1} \\
\dot{x}_{2}
\end{array}\right]=\left[\begin{array}{ll}
A_{11} & A_{12} \\
A_{21} & A_{22}
\end{array}\right]\left[\begin{array}{l}
x_{1} \\
x_{2}
\end{array}\right]+\left[\begin{array}{l}
B_{1} \\
B_{2}
\end{array}\right] u+\left[\begin{array}{l}
C_{1} \\
C_{2}
\end{array}\right] v
$$

where $B_{1}$ is a square non-singular matrix.

It may be possible to choose many alternative sets of variables which lead to $B_{1}$ being square and non-singular.

Then we compute

$$
u=B_{1}^{-1}\left(\dot{x}_{1 \mathrm{~d}}-A_{11} x_{1}-A_{12} x_{2}-C_{1} v\right)
$$

(26) applied to (25) gives

$$
\begin{aligned}
\dot{x}_{1} & =A_{11} x_{1}+A_{12} x_{2}+B_{1} B_{1}^{-1}\left(\dot{x}_{1 \mathrm{~d}}-A_{11} x_{1}-A_{12} x_{2}-C_{1} v\right)+C_{1} v \\
& =\dot{x}_{1 \mathrm{~d}}
\end{aligned}
$$

which shows that we now have control of $x_{1}$.

$$
\begin{aligned}
\dot{x}_{2}= & \left(A_{21}-B_{2} B_{1}^{-1} A_{11}\right) x_{1}+\left(A_{22}-B_{2} B_{1}^{-1} A_{12}\right) x_{2} \\
& +\left(C_{2}-B_{2} B_{1}^{-1} C_{1}\right) v+B_{2} B_{1}^{-1} \dot{x}_{1 \mathrm{~d}}
\end{aligned}
$$

Similar results may be obtained in the non-linear case with

$$
\begin{aligned}
& \dot{x}_{1}=\dot{x}_{1 \mathrm{~d}} \\
& \dot{x}_{2}=\tilde{f}_{2}\left(x, v, \dot{x}_{1 \mathrm{~d}}\right)
\end{aligned}
$$

The changes in $x_{2}$ may be perfectly acceptable if these states are not important to the performance of the process i.e. do not enter explicitly into a measure of the 
performance of the process. If the elements of $x_{2}$ have significance, then the best way to solve the problem is to put them into $x_{1}$. This may be done by choosing another set of elements to compose $x_{1}$ (if possible), or by increasing the number of elements in $u$ and thereby $B_{1}$ and $x_{1}$ in such a way that all the significant states are incorporated in $x_{1}$.

\section{Calculation of control variables from an implicit equation}

If a solution of the form (3) cannot be obtained, a gradient procedure for solving the equation may be used. A criterion may be

$$
\min _{w}\left(\dot{x}_{\mathrm{d}}-f(x, u, \bar{v})\right)^{\mathrm{T}} W\left(\dot{x}_{\mathrm{d}}-f(x, u, \bar{v})\right)
$$

where $W$ is a positive semi-definite weighting matrix.

When only some elements of $\dot{x}_{\mathrm{d}}$ are specified, the rows and columns of $W$ for the unspecified elements are 0 . If $\boldsymbol{f}$ is continuous in $\boldsymbol{u}$ we may differentiate with respect to $u$ and get

$$
\frac{\partial f^{\mathrm{T}}}{\partial u} W\left(\dot{x}_{\mathrm{d}}-f(x, u, v)\right)=0
$$

A possible method of solution is calculating $\boldsymbol{u}$ by integrating a differential equation

$$
\dot{u}=K\left(\dot{x}_{\mathrm{d}}-f(x, u, \bar{v})\right)
$$

where

$$
K=L \frac{\partial f}{\partial u}{ }^{\mathrm{T}} W
$$

$L$ is a non-singular matrix that does not make (33) unstable.

For the following analysis we assume that the process (1) has slow dynamics relative to (33), that $\dot{\boldsymbol{x}}_{\mathrm{d}}$ changes slowly and that we are close to the solution. Then we may linearize (33) around the solution $u_{0}$ and set $\dot{x}_{\mathrm{d}}, \boldsymbol{x}$ and $\bar{v}$ to be constant. Setting $u=u_{0}+\Delta u$ we then get

$$
\Delta \dot{u}=-K \frac{\partial f}{\partial u} \Delta u-\left(\frac{\partial K}{\partial u} \Delta u\right)\left(\dot{x}_{\mathrm{d}}-f\left(x, u_{0}, \bar{v}\right)\right)
$$

Stability and fast convergence of (35) will require its eigenvalues to have large negative real parts, which is also necessary for the above assumptions to be true. If (33) is integrated in a computer, this may put further restrictions on the eigenvalues (example: they cannot have absolute values that are too large).

$K$ should satisfy (34), and this may make it necessary to change $K$ if $\partial f / \partial u$ varies. If only dim $u$ elements of $\dot{x}_{\mathrm{d}}$ are specified, a change in $L$ can compensate for changes in $\partial f / \partial u$ making it possible to use a fixed $K$. The restrictions in the previous paragraph must still be observed.

If the assumptions used to derive (35) are violated, the use of (33) to find the solution may cause instability or difficulties with the convergence. A step change in $\dot{\boldsymbol{x}}_{\mathrm{d}}$ will cause $\boldsymbol{u}$ to be far from the stationary point, and if (1) is very non-linear in $u$, we may get problems with the convergence. Another problem is that we may find a local stationary point rather than a global minimum.

If only dim $\boldsymbol{u}$ elements of $\dot{x}_{\mathrm{d}}$ are specified, $\dot{\boldsymbol{x}}_{\mathrm{d}}$ and $K$ can be calculated independently. For $K$ we can concentrate on the stability and convergence of (33), while the process performance desired is specified through $\dot{\boldsymbol{x}}_{\mathrm{d}}$. 


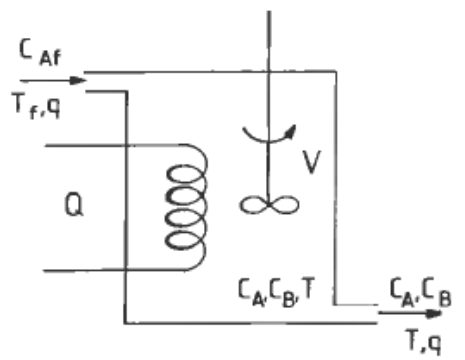

Figure 4. Continuous, stirred tank reactor.

\section{Applications of the non-linear decoupling}

The control method described above could be applied to a number of important industrial processes. Here we will illustrate the method by applying it to the control of the temperature in an exothermic chemical reactor.

\subsection{Chemical reactor with errors in the model}

The assumption is that an irreversible chemical reaction where $A \rightarrow B$ is taking place in a continuous, well-stirred reactor.

The process can be described by the model

$$
\begin{gathered}
\dot{c}_{\mathrm{A}}=\frac{q}{V}\left(c_{\mathrm{Af}}-c_{\mathrm{A}}\right)-r \\
\dot{T}=\frac{q}{V}\left(T_{\mathrm{f}}-T\right)+\frac{1}{C}\left[\left(-\Delta H_{\mathrm{r}}\right) \cdot r \cdot V+Q\right]
\end{gathered}
$$

where $r$ is the rate of the disappearance of $A$

$$
r=k_{\mathrm{C}} \cdot \exp \left(-\left(1 / T-1 / T_{\mathrm{C}}\right) E / R\right) \cdot c_{\mathrm{A}}
$$

Here

$c_{\mathrm{A}}$ concentration of component $A$ in reactor

$c_{\text {Af }}$ concentration of component $A$ in feed

$T$ temperature in reactor

$T_{\mathrm{f}} \quad$ temperature in feed

$Q \quad$ rate of energy supply

$\Delta H_{\mathrm{r}}$ molar heat of reaction

$E \quad$ activation energy

$R$ gas constant

$k_{\mathrm{C}}$ frequency factor

$T_{\mathrm{C}} \quad$ temperature where $r=k_{\mathrm{C}} \cdot c_{\mathrm{A}}$

$C$ heat capacity of reactor content

$V \quad$ volume of vessel

$q \quad$ volumetric feed rate 
If we are concerned with the temperature $T$ of the reactor, we can compute the necessary rate of energy supply $Q$ to arrive at a desired $\dot{T}_{\mathrm{d}}$ from (37)

$$
Q=C \cdot\left[\dot{T}_{\mathrm{d}}-\frac{q}{V}\left(T_{\mathrm{f}}-T\right)\right]+\Delta H_{\mathrm{r}} \cdot r \cdot V
$$

In (39), $\dot{T}_{d}$ is chosen according to (7), but integral action is added in order to handle possible model errors:

$$
\dot{T}_{\mathrm{d}}=K_{\mathrm{p}}^{\mathrm{N}}\left(\left(T_{0}-T\right)+\frac{1}{T_{\mathrm{i}}^{\mathrm{N}}} \int\left(T_{0}-T\right) d t\right)
$$

where

$T_{0} \quad$ temperature set point

$K_{\mathrm{p}}^{\mathrm{N}}$ gain in the PI controller when using non-linear internal decoupling

$T_{i}^{\mathrm{N}}$ integral time in the PI controller when using non-linear internal decoupling

Equations (39) and (40) will change (37) into

$$
\dot{T}=K_{\mathrm{p}}^{\mathrm{N}}\left(\left(T_{0}-T\right)+\frac{1}{T_{\mathrm{i}}^{\mathrm{N}}} \int\left(T_{0}-T\right) d t\right)
$$

Thus, the dynamics of the temperature has become decoupled from the concentration.

We want to compare the performance of this suggested control scheme, (39) and (40), with the performance of a standard PI controller.

To visualize the advantages of the internal non-linear decoupling scheme, we operate the process in such a way that the non-linearities have a significant effect. This is done by changing the set point $T_{0}$ of the temperature around the operating point $T_{\mathrm{Q}}$.

It is well known that an exothermic process can be made stable through increasing the loop gain. To get a fair comparison, however, we tune the parameters of the linear controller so that the loop transfer function of both controller schemes are identical around the operating point, $\left(T_{\mathrm{Q}}, c_{\mathrm{Q}}\right)$.

Using a standard controller, the control variable should be computed as

$$
\begin{aligned}
Q=Q_{\mathrm{s}} & +K_{\mathrm{T}}^{\mathrm{L}}\left(\left(T_{0}-T\right)+\frac{1}{T_{\mathrm{i}}^{\mathrm{L}}} \int\left(T_{0}-T\right) d t\right) \\
& +K_{\mathrm{c}}^{\mathrm{L}}\left(c_{\mathrm{Q}}-c_{\mathrm{A}}\right)
\end{aligned}
$$

where $Q_{s}$ is a stationary value to drive the states close to the operating point. Since the control objective is to keep $T$ as close as possible to $T_{0}$, and since $T_{0}$ varies around $T_{\mathrm{Q}}$, we should calculate $c_{\mathrm{Q}}$ as the stationary value of (36) given $T=T_{\mathrm{Q}}$. In (42)

$K_{\mathrm{T}}^{\mathrm{L}}$ gain in the linear PI controller from a deviation in $T$

$T_{\mathrm{i}}^{\mathrm{L}} \quad$ integral time in the linear PI controller

$K_{\mathrm{c}}^{\mathrm{L}} \quad$ gain in the linear PI controller from a 'deviation' in $c_{\mathrm{A}}$

In addition to studying the response to a changing temperature set point when we have perfect knowledge of the model, we study the same response when two of the 


$$
\begin{array}{ll}
c_{\text {Af }} & =1 \mathrm{~mol} / 1 \\
T_{\mathrm{f}} & =350 \mathrm{~K} \\
E & =8.31 \times 10^{4} \mathrm{~J} / \mathrm{mol} \\
k_{\mathrm{c}} & =1 \mathrm{~min}^{-1} \\
q / V & =1 \mathrm{~min}^{-1} \\
V & =3 \times 10^{3} 1 \\
C & =1 \times 10^{7} \mathrm{~J} / \mathrm{K} \\
\Delta H_{\mathrm{r}} & =-6.71 \times 10^{5} \mathrm{~J} / \mathrm{mol}
\end{array}
$$

Table 1. Parameters used in the simulations

process parameters vary, i.e. $C$ and $E$. To limit the scope of the simulations, we will assume that the rest of the parameters are known exactly, that the feed concentration and temperature are constant, and that the two states are perfectly measurable.

The process has been simulated on a digital computer using Euler integration. The numerical values of the parameters in (36) and (37) are chosen from Perlmutter (1972), except that we have made the process exothermic instead of endothermic.

In all the experiments, we let the parameters in the model of the chemical reactor retain the values given in Table 1 . These values are used to compute the control signal. The values of the parameters $C$ and $E$ in the process itself, are tabulated in Table 2 for the different runs.

In all the experiments, we start at $t=0$ with $T_{0}=380 \mathrm{~K}$ and all states at their stationary values. At $T=0 \cdot 4$, we change $T_{0}$ to $420 \mathrm{~K}$, and at $t=1 \cdot 2$, we change $T_{0}$ back to $380 \mathrm{~K}$ as can be seen in the figures. $T_{\mathrm{Q}}=400 \mathrm{~K}$ is taken to the operating point, and the controller settings are

$$
\begin{array}{ll}
K_{\mathrm{T}}^{\mathrm{L}}=3 \times 10^{8} & T_{\mathrm{i}}^{\mathrm{L}}=1 \cdot 0 \\
K_{\mathrm{p}}^{\mathrm{N}}=30 & T_{\mathrm{i}}^{\mathrm{N}}=1 \cdot 0
\end{array}
$$

Figure 5 shows that the response in $T$ to a change in $T_{0}$ is much better when using the non-linear controller, than when the linear PI controller is ulilized. Figures 8-11 also show that the response is much less sensitive to model errors when using the non-linear controller rather than the linear PI controller. It can be argued that an

\begin{tabular}{rcccc}
\hline $\begin{array}{c}\text { Sim. } \\
\text { no. }\end{array}$ & $\begin{array}{c}E(\mathrm{~J} / \mathrm{mol}) \text { in } \\
\text { the process }\end{array}$ & $\begin{array}{c}C(\mathrm{~J} / \mathrm{K}) \text { in } \\
\text { the process }\end{array}$ & Cs $\dagger$ & $\begin{array}{c}\text { Result } \\
\text { in Figure }\end{array}$ \\
\hline 1 & $8.31 \times 10^{4}$ & $1 \times 10^{7}$ & $\mathrm{~L}$ & $5,6,7,8,10$ \\
2 & $8.31 \times 10^{4}$ & $1 \times 10^{7}$ & $\mathrm{~N}$ & $5,6,7,9,11$ \\
3 & $8.31 \times 10^{4}$ & $0.8 \times 10^{7}$ & $\mathrm{~L}$ & 8 \\
4 & $8.31 \times 10^{4}$ & $0.8 \times 10^{7}$ & $\mathrm{~N}$ & 9 \\
5 & $8.31 \times 10^{4}$ & $1.5 \times 10^{7}$ & $\mathrm{~L}$ & 8 \\
6 & $8.31 \times 10^{4}$ & $1.5 \times 10^{7}$ & $\mathrm{~N}$ & 9 \\
7 & $7.5 \times 10^{4}$ & $1 \times 10^{7}$ & $\mathrm{~L}$ & 10 \\
8 & $7.5 \times 10^{4}$ & $1 \times 10^{7}$ & $\mathrm{~N}$ & 11 \\
9 & $15 \times 10^{4}$ & $1 \times 10^{7}$ & $\mathrm{~L}$ & 10 \\
10 & $15 \times 10^{4}$ & $1 \times 10^{7}$ & $\mathrm{~N}$ & 11 \\
\hline
\end{tabular}

† Controller scheme: $L$ is a standard PI controller, $N$ is PI controller with internal nonlinear decoupling.

Table 2. Values used for the varying process parameters. 


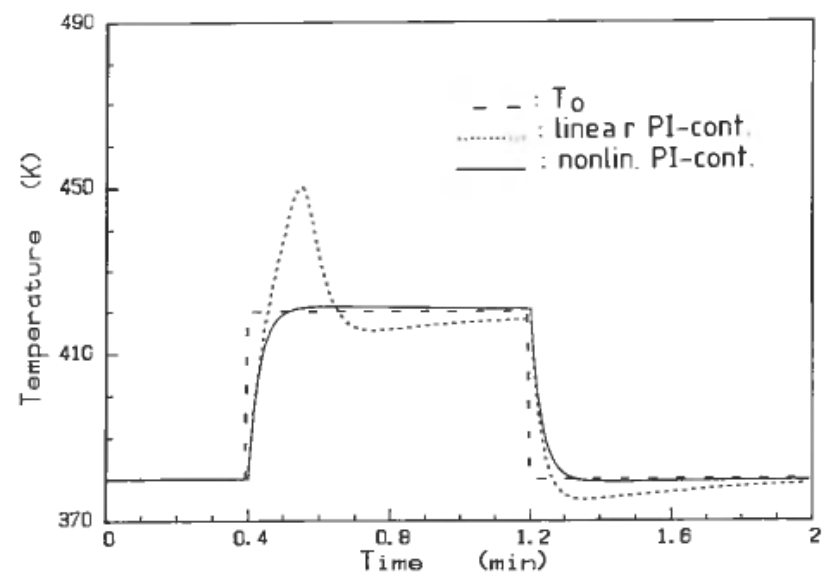

Figure 5. $T$ in the case of perfectly known process.

increase in the loop gain will give better performance and less sensitivity to model errors for the linear PI controller. But the same argument is valid for the internal non-linear decoupling scheme.

The large overshoot in $T$ using a standard PI controller is mainly due to the integral action. A better implemented integral action or feedforward from the set

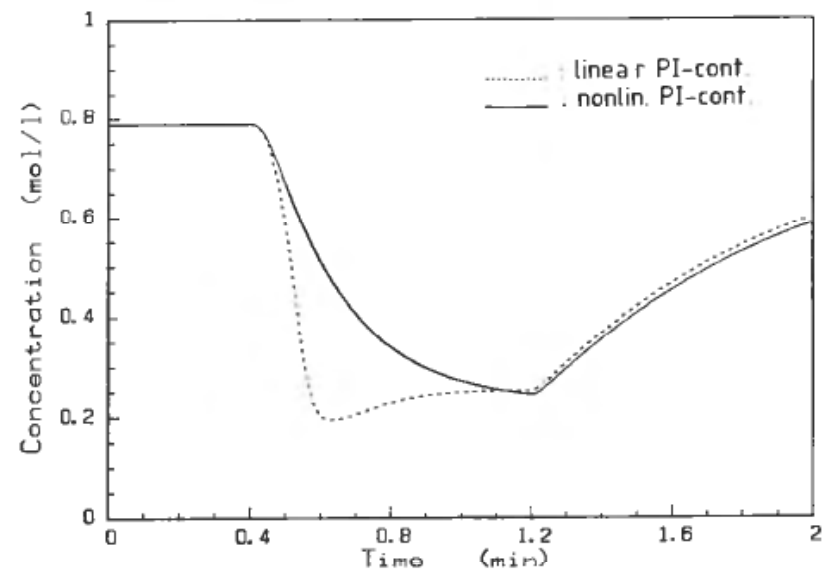

Figure 6. $c_{\mathrm{A}}$, same case as Figure 5 .

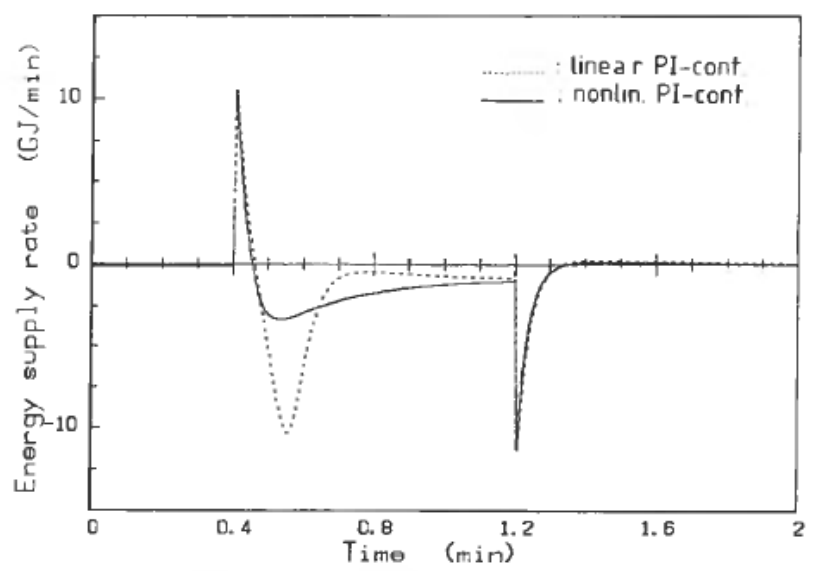

Figure 7. $Q$, same case as Figure 5 . 


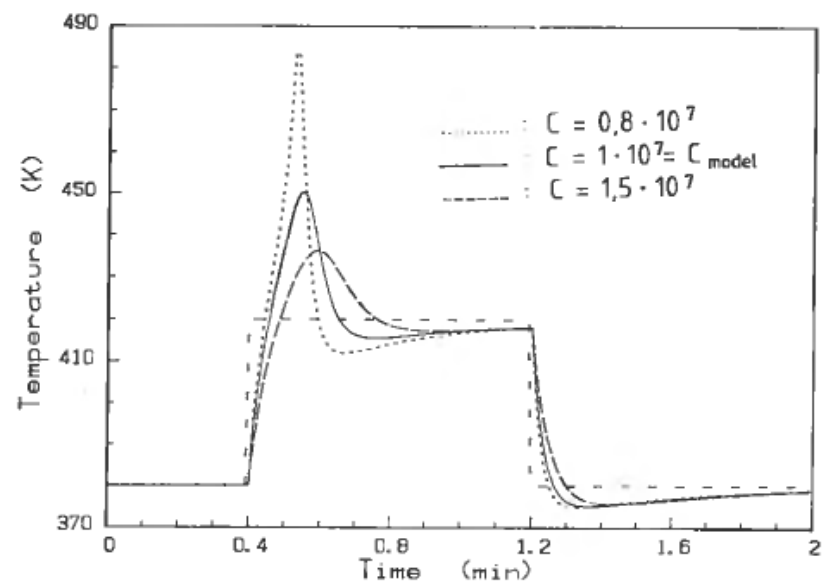

Figure 8. T, effect of $C$ different in model and process when using linear PI controller. (to be compared with Figure 9.)

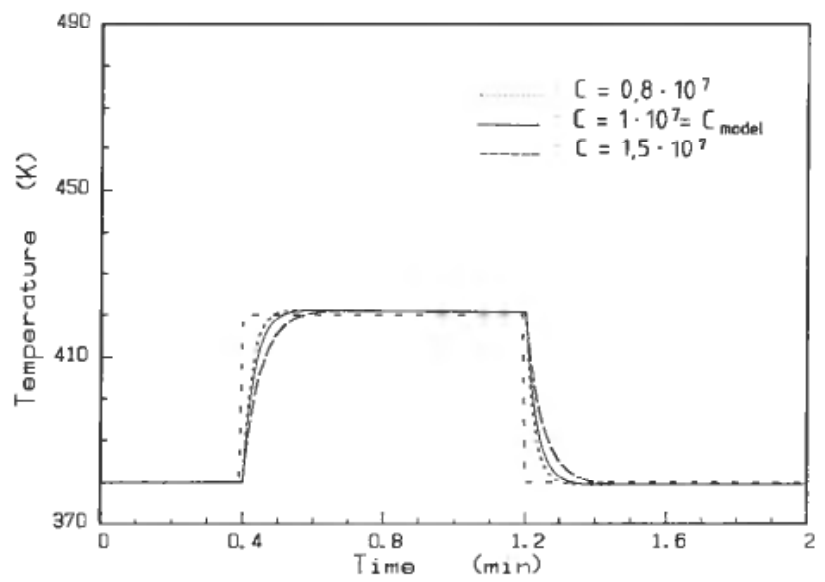

Figure 9. $T$, effect of $C$ different in model and process when using internal non-linear decoupling. (to be compared with Figure 8.)

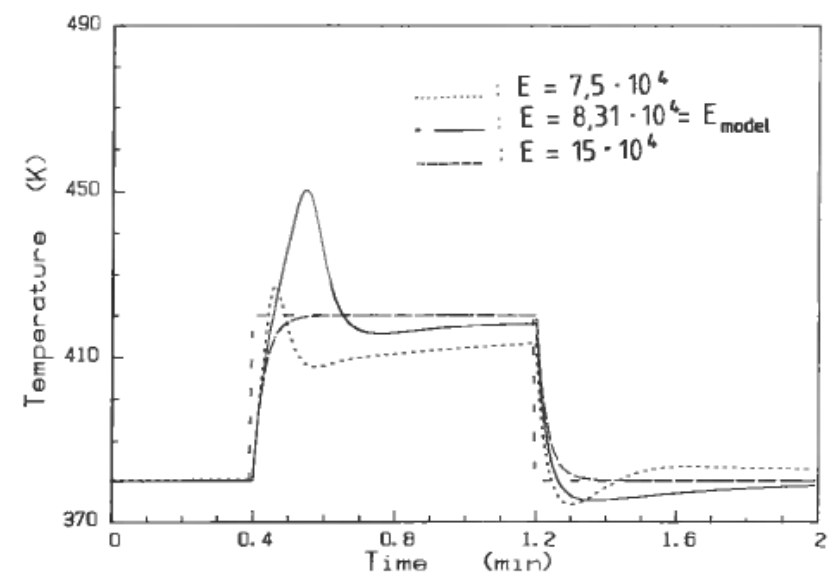

Figure 10. T, effect of $E$ different in model and process when using linear PI controller. (to be compared with Figure 11.) 


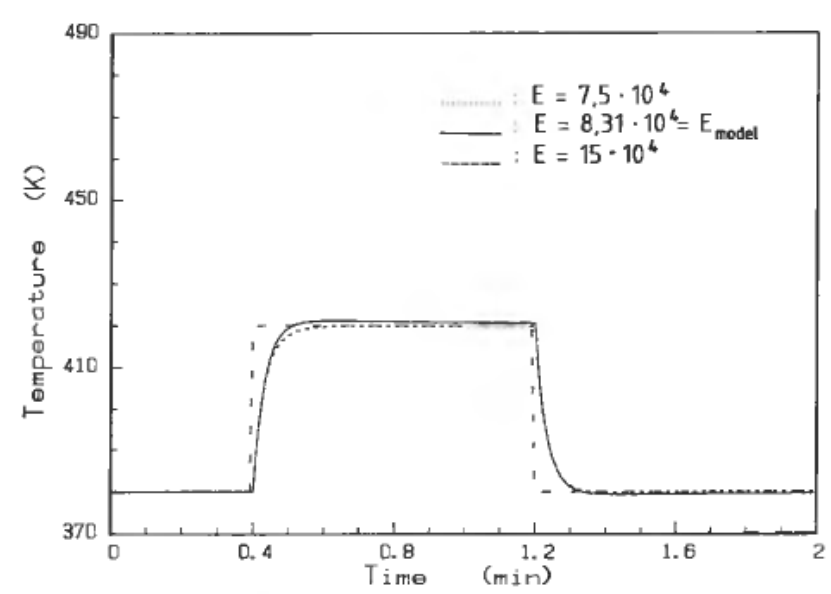

Figure 11. $T$, effect of $E$ different in model and process when using internal non-linear decoupling. The case when $E=15 \times 10^{4}$ is hidden behind $E=8.31 \times 10^{4}$. (to be compared with Figure 10.)

point can partly solve this problem. This will however make the controller scheme rather complicated compared to the simple scheme of internal non-linear decoupling, and still give inferior performance.

\section{Conclusion}

A simple method has been investigated for the total or partial removal of the effect of non-linear process phenomena in feedback control systems. The method is based on the computation of control variables which will yield prescribed rates of change in the process states. Simulations have shown that this control scheme gives better performance than that given by a standard PI controller. This is so with respect to both transient behaviour and robustness to parameter errors in the model. Furthermore, it is shown by analysis that the specified states can be made to approach their set points infinitely fast without giving stationary deviation, if we use infinitely large feedback gains.

In practice, however, it is not feasible to employ very large gains, since when the system becomes fast, effects which were neglected in the modeling phase will become significant. If these effects are to be taken into account it is necessary to increase the dimension of the model. A simple example of this is neglecting a transport delay.

As this non-linear decoupling algorithm normally has to be implemented on a digital computer, the sampling rate will also effectively limit the possible bandwidth of the process.

\section{REFERENCES}

Hunt, L. R. Su R. and Meyer, G. (1983). Global transformations of non-linear systems. I.E.E.E. Trans. Aut. Cont., 28, 24-31.

Juba, M. R. and Hamer, J. W. (1986). Progress and challenges in batch process control. 3rd Int. Conf. Proc. Cont. (CPC III), Asilomar Calif. Jan 1986.

PAUL, R. C. (1972). Modeling trajectory calculation and servoing of a computer-controlled arm. Ph.D. thesis, Stanford Art. Int. Lab, Stanford Univ., Stanford, California.

Perlmutter, D. D. (1972). Example 2.3 in Stability of chemical reactors, (Prentice-Hall, Inc; Englewood Cliffs, New Jersey). 